\title{
In search of an adequate educational policy for foreign language teaching in Slovakia
}

\section{[A la recherche d'une politique educative adequate pour l'enseignement des langues en Slovaquie ?]}

\author{
Maria Rostekova
}

DOI: 10.18355/XL.2019.12.01XL.06

\begin{abstract}
This contribution addresses the issue of foreign language teaching as a component of the Slovak educational policy. A good command of several languages is indeed crucial for the internationalization of education as well as for keeping countries competitive on a European or global scale. While many countries are implementing strategies focused on education, we are somewhat disappointed by recent developments that we have observed in Slovakia regarding how foreign languages are taught and learned. To better grasp that situation, we need to examine both the criteria that motivate learners to choose one or more foreign languages and which languages are offered by the Slovak education system. For that purpose, we have developed a quantitative survey as a pre-research step before embarking upon more extensive research. We need to analyze several theoretical notions that are essential for understanding our objective, particularly soft power, education policy and social representations of foreign languages. Our ambition is to avoid relying on pedagogical considerations that underpin most approaches in this field. Rather, we intend to focus on the political, historical, and sociolinguistic aspects of foreign language education.

Key words: educational policy, foreign language teaching, soft power, social representations of languages

\section{Résumé}

Cette contribution aborde la question de l'enseignement des langues étrangères comme composante de la politique éducative en Slovaquie. Une bonne maîtrise de plusieurs langues s'avère en effet cruciale pour l'internationalisation de l'enseignement ainsi que pour la compétitivité des pays à l'échelle européenne ou mondiale. Si les gouvernements de nombreux États mettent en place des stratégies dédiées à l'éducation, les évolutions que nous avons pu récemment observer en Slovaquie dans les domaines de l'enseignement et de l'apprentissage des langues ne sont pas toujours satisfaisantes. Pour les saisir correctement, il convient d'observer les critères ayant motivé les apprenants à choisir une ou plusieurs langues vivantes tout en analysant l'offre de langues proposée par le système éducatif slovaque. Nous avons élaboré dans ce but une enquête quantitative que nous considérons comme une sorte de prérecherche, cruciale pour se familiariser de la façon la plus complète possible avec le sujet avant d'entamer des recherches de plus grande ampleur. Plusieurs notions théoriques indispensables pour la compréhension de l'objectif seront analysées, notamment celles du soft power, de la politique éducative et des représentations sociales des langues - notre ambition étant de nous démarquer des démarches habituelles en la matière, basées la plupart du temps sur des considérations pédagogiques pour nous intéresser en priorité à des aspects politiques, historiques et sociolinguistiques.
\end{abstract}

Mots-clés : politique éducative, enseignement des langues étrangères, soft power, représentations sociales des langues 


\section{Introduction}

L'enseignement des langues étrangères constitue l'une des préoccupations majeures de tout système éducatif européen, car ce champ se distingue de celui des autres matières enseignées. Nous avons pu constater un accroissement de sa reconnaissance en 2004 après l'élargissement de l'Union européenne - l'arrivée de 10 nouveaux États membres occasionnant une augmentation sans précédent du nombre de langues officielles. La politique de multilinguisme de l'UE poursuit deux objectifs majeurs : le premier est de protéger la grande diversité linguistique de l'Europe et le second d'encourager l'apprentissage des langues vivantes. Si cela a abouti dans certains pays à approfondir une tendance déjà présente depuis longtemps et ancrée dans les conditions historiques particulières de ces États, comme par exemple en Slovaquie (Hanesova, 2015), les évolutions observées dans d'autres pays peuvent être interprétées comme le reflet de mutations sociales, politiques et culturelles d'ampleur plus significative.

L'enseignement des langues étrangères s'est alors inscrit plus profondément dans les systèmes éducatifs à cause de cette dynamique européenne. La connaissance de plusieurs langues est devenue, pour les Européens, une exigence dans la vie quotidienne comme dans la vie professionnelle, notamment pour l'ouverture au monde (Schmitt, 2014), le dialogue interculturel (Meskova, 2016; Roskova, 2006 ; Zazrivcova, 2014), la tolérance (Badinska, 2016 ; Molnarova, 2015), la mobilité au cours de la formation (Gura - Rostekova, 2015 ; Rizekova, 2018) ou encore pour le marché de l'emploi (Palova - Zelenakova, 2018). Cependant, ce plurilinguisme qui fait partie de l'Europe, aussi bien à l'échelle du continent qu'au niveau national et régional, soulève régulièrement des questions assez complexes auxquelles s'ajoute celle de l'organisation de cette diversité des langues. De nouvelles approches du plurilinguisme en Europe, telle que l'intercompréhension (Kubekova, 2017; Chovancova, 2008) ou encore la mise en avant de l'intelligence interculturelle (Bencekova, 2016) semblent apporter des réponses plus satisfaisantes à certains des défis soulevés.

Les travaux de chercheurs slovaques relatifs aux aspects susmentionnés sont nombreux. Cependant, la présente contribution a pour ambition de se concentrer sur un autre aspect très important lié aux langues, ou plus précisément à l'internationalisation de l'enseignement (pour laquelle une bonne maîtrise des langues s'avère cruciale) : celui de la compétitivité de l'UE et/ou des États membres au niveau mondial. Dans ce contexte, l'intérêt de chercheurs de plusieurs champs disciplinaires se porte sur la qualité et l'attractivité des systèmes éducatifs des pays en tant qu'éléments-clés du soft power ou objet des stratégies mises en place par des gouvernements. En tant qu'enseignante de français et spécialiste des relations internationales, nous participons actuellement au projet de recherche « Le concept de "soft power" dans le contexte d'un environnement international en mutation et son potentiel pour les stratégies des petits États » (VEGA n 1/0949/17) dans le cadre duquel nous nous intéressons au rôle de l'éducation comme outil de positionnement des pays sur le plan international.

Si un grand nombre de pays ne ménagent pas leurs efforts pour rendre leurs systèmes compétitifs sur le plan international, de nombreuses voix se sont élevées dernièrement en Slovaquie afin de pointer l'état insatisfaisant dans lequel se trouvent les établissements scolaires du pays. Les statistiques mondiales et européennes lors de l'évaluation des élèves, étudiants et universités montrent en effet clairement que notre système éducatif décline progressivement. Les critiques visent également l'enseignement des langues, notamment la baisse des compétences langagières des 
jeunes Slovaques, mais aussi l'affaiblissement de leur intérêt pour l'apprentissage des langues vivantes, constat que nous avons également pu faire lors de notre expérience pédagogique. Nous avons donc décidé, dans le cadre du projet mentionné précédemment, de porter notre attention sur la politique éducative slovaque en matière d'enseignement des langues étrangères : des performances langagières suffisantes étant en effet indispensables pour l'internationalisation de l'enseignement supérieur, ainsi que pour l'insertion des jeunes diplômés slovaques sur le marché internationalisé de travail.

\section{L'éducation comme outil par excellence du bon positionnement d'un pays à l'échelle internationale}

Le concept de soft power, que l'on peut traduire en français comme "manière douce» ou "pouvoir de convaincre » est un concept développé en 1990 par le théoricien politique Joseph Nye de l'Université de Harvard pour décrire la capacité d'un État à attirer plutôt que de contraindre ou d'utiliser la force militaire, économique et politique comme moyen de persuasion (Nye, 2009: 8) ${ }^{1}$. Ce pouvoir tire parti de l'attractivité des valeurs proposées, des institutions et s'appuie sur la capacité de créer des normes internationales et des normes de comportement. La culture, l'éducation et la recherche y deviennent des instruments parmi les plus efficaces : seul un système éducatif développé, répondant aux exigences d'une économie innovante de haute technologie et intégré dans l'espace éducatif et scientifique international, peut devenir l'un des principaux avantages concurrentiels de l'État moderne face à la concurrence mondiale des esprits et attirer le plus grand nombre d'étudiants étrangers talentueux (Cowan - Arsenault, 2008:10).

De nos jours, le nombre de pays considérant l'éducation comme le meilleur moyen de promouvoir leurs intérêts nationaux sur la scène mondiale est en augmentation. Cependant, l'attractivité exercée par un pays sur un autre concerne principalement les États les plus développés ou les plus puissants au monde (Gura, 2018 ; Bolecekova, 2018). Mais cela ne signifie pas qu'une éducation de qualité ne doive pas concentrer les intérêts de petits pays, tels que la Slovaquie (Terem, 2011 ; Terem - Cajka Rysova, 2015). Définie par les politiques publiques, l'éducation représente l'un des principaux piliers du maintien et du développement du bien-être de chaque État - il s'agit de l'un des facteurs les plus importants de la croissance économique. Le niveau d'éducation se reflète, entre autres, dans la compétitivité de l'économie, dans la croissance de la productivité du travail ou dans la réduction du chômage et dans la capacité de l'individu à se positionner sur le marché de l'emploi.

En dépit d'un potentiel de soft power relativement limité, les petits pays de l'UE peuvent désormais s'attaquer collectivement aux problèmes politiques et économiques posés par le processus de mondialisation (Kalicky - Lasicova, 2017 ; Rosputinsky, 2016 ; Vlcek, 2018) et l'éducation offre des solutions concrètes dans cette optique. Même si le traité de Rome ne prévoyait pas de compétence commune en matière d'éducation et de formation, des termes liés à ces domaines sont progressivement apparus dans le vocabulaire européen, notamment avec le traité de Maastricht. Si la compétence de chaque État membre reste prépondérante de nos jours, l'Union européenne favorise néanmoins la coopération entre États en matière d'enseignement, complète leur action et encourage l'émergence de pratiques innovantes, en respectant pleinement les orientations souhaitées par les pays en matière de contenu de

\footnotetext{
${ }^{1}$ Parallèlement, le soft power s'oppose au « hard power » qui implique le recours à la force et à la contrainte militaires.
} 
l'enseignement et d'organisation du système éducatif tout en tenant compte de la diversité culturelle et linguistique de chacun.

Des réformes modifient les systèmes scolaires et universitaires partout en Europe. Ces changements sont dûs en particulier aux nouveaux défis posés, comme par exemple l'accélération du progrès technologique et l'impact du numérique, mais aussi l'internationalisation croissante de la vie économique et sociale qui est au cœur de notre analyse. Ces réformes n'interviennent toutefois pas au même rythme selon les pays. La Finlande, par exemple, considérée comme un modèle de performance au niveau européen dans toutes les enquêtes sur les acquis des élèves a néanmoins entrepris en 2013 une réforme fondamentale, malgré les succès déjà obtenus sur le plan international. ${ }^{2}$

Selon les discours politiques, les réformes slovaques prennent en compte les tendances observées dans le contexte européen. En ce qui concerne l'enseignement et l'apprentissage des langues étrangères, la Slovaquie a participé assez activement à divers programmes de soutien (Kubekova, 2018). L'étape la plus importante en ce sens a été l'année 2001 qui a vu l'adoption du Programme du Millénaire. L'un des principaux objectifs de ce programme était l'introduction d'une langue étrangère obligatoire et d'une deuxième langue optionnelle. Cet objectif a été atteint au cours de l'année scolaire 2011-2012, lorsque l'anglais est devenu la première langue étrangère obligatoire à partir de la troisième année du primaire (élèves âgés de 8 ans) et que la deuxième langue étrangère est devenue obligatoire entre la sixième et la neuvième année du primaire (élèves âgés de 12 à 15 ans). ${ }^{3}$ Une adaptation ultérieure a instauré l'enseignement de la deuxième langue étrangère à partir de la première année du deuxième cycle du primaire (élèves âgés de 11 ans). Cette expérimentation a pris fin en 2015 , donnant à la deuxième langue étrangère le statut de matière facultative ${ }^{4}$.

Nous observons actuellement des évolutions peu satisfaisantes, notamment une baisse $\mathrm{du}$ niveau en langues étrangères des candidats aux études universitaires, mais également des diplômés entrant sur le marché du travail. Cette évolution négative touche également le nombre d'apprenants de certaines langues, bien que celles-ci soient ancrées depuis des décennies dans le système éducatif slovaque - c'est notamment le cas du français et de l'allemand. L'unique augmentation significative du nombre d'apprenants concerne la langue russe.

Cette évolution nous paraît surprenante, voire paradoxale. Tout d'abord en raison de l'importante présence économique allemande et française sur le territoire slovaque réalité qui se traduit dans l'offre de postes sur le marché slovaque du travail. La bonne maîtrise de ces deux langues est donc indispensable et de nombreux employeurs confient aujourd'hui rencontrer des difficultés liées à l'embauche de candidats ayant un niveau de langue correspondant à leurs attentes (de $\mathrm{B} 1$ à $\mathrm{C} 1 \mathrm{~d}$ 'après le CECRL). Nous ne pouvons non plus négliger les relations bilatérales étroites de la France et de l'Allemagne avec la République slovaque. Pour la première citée, cette coopération

\footnotetext{
${ }^{2}$ L'objectif principal est l'abandon d'une partie des enseignements par discipline au profit d'enseignements par sujets ou par thèmes.

${ }^{3}$ L'école primaire en Slovaquie regroupe les enfants âgés de 6 à15 ans et se compose de deux niveaux : le premier dure 4 ans et le second est 5 ans.

${ }^{4}$ Le terme « optionnel » se réfère au fait de choisir entre deux ou plusieurs options (dans notre cas choisir une langue parmi plusieurs proposées). Une matière facultative est en revanche non-obligatoire.
}

XLinguae, Volume 12 Issue 1XL, January 2019, ISSN 1337-8384, eISSN 2453-711X 
est encadrée par un accord de partenariat stratégique, signé à Paris en 2008 et renouvelé pour les années 2013-2018 - le volet éducatif y occupe une place importante.

Dans ce contexte, il convient de souligner l'importance des programmes d'études conjoints ou doubles, proposés par plusieurs universités slovaques en coopération avec des universités françaises. Le savoir-faire français dans le domaine de la professionnalisation de l'enseignement supérieur est tout aussi important: l'expérience française dans le domaine de la diplomatie culturelle est particulière et ne connaît pas, selon nous, d'équivalent comparable au niveau mondial (Gura, 2011). La création en 2011 à l'Université Matej Bel de Banská Bystrica de l'Institut universitaire franco-slovaque (IUFS) - institution visant à assurer le partage du savoir-faire français à l'échelle slovaque - démontre également l'importance de la coopération franco-slovaque (Rostekova, 2018).

Expliquer les causes des changements observés est une tâche complexe qui ne facilite pas la formulation de conclusions ou de projections pour l'avenir. La problématique soulevée est assez délicate: pour l'appréhender correctement, il faut d'abord comprendre comment s'opèrent les choix et de quelle façon est constituée l'offre de langues au sein du système éducatif slovaque. Cette analyse permettra ensuite de repérer les facteurs ayant considérablement changé la donne en l'espace de quelques années.

Nous avons donc décidé dans ce but d'effectuer des recherches centrées sur l'offre et le choix de langues vivantes du système éducatif slovaque, ainsi que sur le rôle des divers acteurs intervenant au moment de ces choix. L'enquête présentée dans le cadre de cette contribution ne constitue cependant qu'une étape préparatoire, une sorte de prérecherche primordiale afin de nous familiariser de la façon la plus complète possible avec notre sujet d'étude. Avant d'observer les différents résultats obtenus lors de nos travaux, nous allons nous pencher sur certaines notions théoriques, préalables à la compréhension de l'objectif de notre recherche et à l'exploitation des données recueillies, notamment les concepts de politique éducative et de représentations sociales des langues.

\section{Quelle politique pour l'enseignement des langues étrangères ?}

Si le terme de politique éducative (ci-après : «P.É. ») reste fréquemment utilisé par les spécialistes et le grand public partout dans le monde, il n'est cependant pas délimité de façon suffisante ou catégorique (Ball, 1993). Des sources en langue étrangère font état de débats autour de la signification de ce concept. Nous nous permettons néanmoins d'affirmer que le caractère étendu et approfondi de ces discussions aboutit à une confusion substantielle. La situation inverse est constatée en Slovaquie - ainsi que chez son voisin tchèque (Vesely, 2013) : si des définitions de ce qu'est la politique éducative existent dans ces deux pays, le débat critique visant à la définir précisément est absent ou s'est avéré insuffisant. La P.É. est généralement délimitée par une définition générale qui est ensuite maniée comme si elle était indiscutable.

Il est également important d'attirer l'attention sur l'aspect terminologique du sujet. Le français, le slovaque et la majorité des langues ne disposent que d'un seul mot pour parler de politique tandis que l'anglais peut nuancer à l'aide des termes "polity », " politics » et "policy » (Fiala - Schubert, 2000). "Polity » désigne un règlement politique existant ou exigé. Il s'agit, tout à la fois, de la délimitation de l'espace dans 
lequel se joue la politique et de la structure de cet espace ${ }^{5}$. «Politics » représente le processus dynamique de création de la politique dans lequel se rencontrent les intérêts et les procédés les plus divers qui s'imposent ou non sur la base d'un conflit ou d'un consensus. Le terme "policy» qualifie pour sa part le contenu matériel de la politique.

Ces termes présentent les trois dimensions de la politique qui sont interconnectées entre elles. La P.É. se déroule toujours dans un certain cadre institutionnel définissant des règles formelles et informelles de fonctionnement, liées en particulier à l'acquisition et à la distribution de compétences et de responsabilités. La situation réelle de chaque État diffère par exemple sensiblement en fonction de la manière dont sont élus ou choisis les décideurs - outre les enseignants et directeurs d'établissement, cela concerne également les fonctionnaires ainsi que le personnel politique. Si la plupart des systèmes confient la question de l'éducation à des hommes et femmes politiques "multifonctions » élus à l'issue de scrutins nationaux ou régionaux, le pouvoir de décision peut relever localement de conseils auxquels candidatent des politiciens spécialistes des questions éducatives (anciens enseignants ou chefs d'établissement). La concentration du pouvoir et de la responsabilité constitue également une question importante.

Les recherches publiées à l'étranger démontrent que le non-éclaircissement du concept de P.É. se manifeste de différentes façons. Plusieurs spécialistes se sont notamment demandé pourquoi les élèves de certains États obtenaient de bien meilleurs résultats scolaires que ceux d'autres pays. Si de nombreux facteurs pesant sur les résultats ne peuvent être influencés directement par une décision politique (c'est le cas notamment de la culture et de la valeur de l'éducation dans une société), la plupart des auteurs supposent qu'il est possible d'améliorer sensiblement les performances en appliquant une politique adéquate en matière d'éducation (Whelan, 2009 ; Levin, 2008).

Tous les aspects mentionnés dépassent le cadre de la pédagogie et font (ou devraient faire) l'objet de recherches en science politique, le cas échéant en économie, sociologie, etc. (Vesely, 2013). La vision de l'orientation sur le long terme de l'éducation en Slovaquie étant principalement due à l'ajustement entre "polity » et " politics », nous considérons comme pertinent le fait que la pédagogie s'intéresse à ces aspects ou, tout du moins, prenne en compte les études réalisées dans ces domaines afin de les traiter, soutenir ou commenter.

En observant la réflexion sur la P.É. menée en Slovaquie, nous constatons que les questions de "polity » et de «politics » dans l'éducation font l'objet d'une attention relativement faible en dépit des modifications essentielles intervenues après la chute du mur de Berlin. Les changements politico-sociaux et socio-économiques d'après novembre 1989 ont logiquement imposé des mutations essentielles touchant non

\footnotetext{
${ }^{5}$ Il convient de remarquer que le mot «polity » reste peu usité dans la littérature spécialisée par rapport au terme "governance ». Les deux concepts ont vu le jour dans des contextes différents et il est impossible de les considérer comme des synonymes. «Polity » se contente la plupart du temps de définir « uniquement» les règles $\mathrm{du}$ jeu de la politique tandis que la définition de la gouvernance s'avère plus large et englobe tous les modes de régulation de la société et de l'économie (incluant donc les acteurs "non-politiques »). Les deux termes se chevauchent cependant de façon importante.
}

XLinguae, Volume 12 Issue 1XL, January 2019, ISSN 1337-8384, eISSN 2453-711X 
seulement la loi, mais aussi l'organisation de la pédagogie et surtout l'idéologie de celle-ci dans le domaine de l'éducation et de la gestion de cette dernière. (Vzdelávací systém v SR, 2018). Cette transformation tenait compte, bien évidemment, des textes et recommandations émanant d'institutions internationales régissant la formation et l'éducation (UNESCO, OCDE, Déclaration de Bologne, etc.).

L'étendue et la complexité de ce cadre politico-social et socio-pédagogique expliquent pourquoi ces changements n'ont eu lieu que de façon progressive. Il est également nécessaire de souligner que les propositions présentées et adoptées dans les années 1990 n'ont été ni approuvées ni appliquées. ${ }^{6}$ En 2017, de larges débats publics réunissant des spécialistes ont mené à la proposition d'un programme national pour le développement de la formation et de l'éducation intitulé La Slovaquie qui apprend, rédigé par le ministère de l'Éducation, de la Recherche scientifique et des Sports de la République slovaque. Le grand public a pu participer à la discussion en transmettant ses remarques ou suggestions par le biais du portail internet du ministère. L'objectif déclaré était la création commune d'un document intemporel et apolitique reflétant les besoins des citoyens slovaques. Des représentants d'associations slovaques d'enseignants de langues étrangères ont également formulé des commentaires relatifs à l'enseignement des langues vivantes. Cette phase d'observation achevée, une équipe d'auteurs a ensuite évalué et retranscrit les suggestions les plus significatives. Le rapport n'a cependant pas trouvé grâce aux yeux du ministère qui a repoussé l'adoption du nouveau programme national.

L'expérience slovaque permet néanmoins d'observer que les nombreux différends présentés comme des «querelles de spécialistes» sont le reflet de conflits plus profonds entre des acteurs aux perspectives et intérêts divers. La question du pouvoir est alors clairement indissociable de la P.É. (Fowler, 2004). Il n'est d'ailleurs pas fortuit que tous les travaux scientifiques d'importance relatifs à la P.É. publiés récemment par des spécialistes de nombreux pays soient consacrés, entre autres, à l'utilisation du pouvoir par les différents acteurs et à la rencontre d'intérêts divers.

Pour saisir correctement les évolutions défavorables liées à l'enseignement des langues en Slovaquie mentionnées précédemment, il est indispensable de comprendre ce que signifie précisément le concept de politique éducative dans notre pays, en particulier pour l'enseignement des langues: Désigne-t-il uniquement une décision politique ou inclue-t-il un texte adopté ? S'agit-il alors de l'application de cette décision ou de ce texte? Ou encore, à qui incombe la responsabilité de créer cette politique éducative? Nous ne mentionnons ici que quelques-unes des nombreuses questions qui pourraient être soulevées par l'utilisation de la notion de P.É. sans avoir l'ambition de donner des réponses définitives dans le cadre de cette contribution. Il s'agit plutôt de montrer qu'il est possible de s'écarter des procédés habituels basés sur des considérations pédagogiques, pour favoriser des démarches politiques, historiques ou sociolinguistiques. L'application d'une telle approche à la question de l'enseignement des langues étrangères pourrait constituer un développement nouveau en Slovaquie.

\footnotetext{
${ }^{6}$ Problème supplémentaire : le changement de politique éducative survenue lors de la transformation de l'ensemble de la société dans les années 1990 n'a pas bénéficié d'un climat socio-économique favorable. On a assisté durant cette période a une chute de la productivité économique et a un chômage important, y compris chez les diplômés, ce qui a occasionné une baisse de la demande de personnel qualifié ainsi qu'une diminution de la fonction sociale de la formation et de l'éducation.
} 


\section{Le choix des langues vivantes et la constitution de leur offre dans les écoles slovaques}

Étant donné leur âge, il va de soi que les enfants du niveau primaire ne choisissent pas les langues étrangères qu'ils étudient. Ce choix relève d'un contexte plus large, surtout familial, lui-même influencé par d'autres facteurs : culturel, géographique, etc. (Deregnaucourt, 2011). Si les lycéens semblent être un peu plus libres par rapport aux élèves du primaire, l'influence direct ou indirecte du contexte familial est également significative.

Selon la littérature spécialisée, cette problématique ne devrait pas être dissociée des représentations sociales des langues, car celles-ci se trouvent au cœur des choix de langues vivantes dans les systèmes scolaires. Pour certains auteurs, dont V. Derégnaucourt (2011), elles y sont absolument déterminantes. Les représentations que les locuteurs se font des langues, de leurs normes, de leurs caractéristiques ou de leurs statuts au regard d'autres langues influencent les procédures et les stratégies qu'ils développent et mettent en œuvre pour les apprendre et les utiliser (Dabene 1997). Ces représentations engendrent des attitudes vis-à-vis des langues qui ne sont pas uniquement liées à l'expérience personnelle et sont souvent déterminées par d'autres éléments, car l'absence de relation personnelle à un objet n'empêche pas d'avoir une attitude, positive ou négative, à son égard. Il s'agit de représentations sociales transmises par la famille, les pairs, l'école ou par un message plus global envoyé par la société.

Les travaux de L. Dabène $(1994,1997)$, qui s'est beaucoup consacrée à la problématique de l'image des langues, semblent être des plus pertinents pour l'objectif de notre travail. Dans sa théorie, l'auteure évoque les statuts formels et informels des langues, c'est-à-dire leur pratique concrète et le discours pouvant être tenu à leur sujet. Le statut informel, implicite ou explicite, donne à la langue une certaine valeur. Selon L. Dabène, cinq critères sont importants pour déterminer la valeur d'une langue dans le cadre de ce statut : économique, social, culturel, épistémique et affectif.

Le critère économique est lié à la valeur de la langue sur le marché du travail. Ce « pouvoir économique " de la langue apporté à ses locuteurs constitue un aspect important de sa diffusion. Le critère social relève de l'image sociale véhiculée par ses locuteurs, de leur statut dans la société. La maîtrise de cette langue prend alors l'image d'un facteur d'ascension sociale ou est perçue au contraire comme un frein à une certaine élévation. Le critère culturel porte sur la richesse culturelle associée à la langue, ainsi que sur sa valeur esthétique. Le critère épistémique concerne la structure-même de la langue, notamment d'un point de vue grammatical et lexical : plus la langue est jugée difficile, plus sa valeur est importante. Enfin, le critère affectif fait appel aux préjugés, favorables ou défavorables, accompagnant la langue. Ces préjugés sont modulés par «les aléas de l'histoire, [l']émergence des langues sur la scène internationale et [les] relations harmonieuses ou conflictuelles entre les pays où on les parle » (Deregnaucourt, 2011).

Nous pouvons aussi mentionner d'autres critères en plus de ceux énumérés par L. Dabène, comme la proximité géographique, évoquée par exemple dans les travaux de A. Mondavio (1997). Dans ce sens, les langues les plus proches géographiquement seraient jugées comme plus utiles, et leur apprentissage serait donc privilégié par les élèves et leurs parents, notamment dans les zones frontalières. L'auteure fait aussi référence à la notion de plaisir qui doit également être prise en considération. Une langue peut être choisie parce que son apprentissage peut être source de plaisir,

XLinguae, Volume 12 Issue 1XL, January 2019, ISSN 1337-8384, eISSN 2453-711X 
qu'elle est agréable à entendre ou à parler, ou parce que la connaître ouvre certaines perspectives dans le domaine des loisirs.

Nous tenons également à mentionner un élément non négligeable pouvant influencer plus ou moins inconsciemment les élèves et/ou leurs parents : les politiques linguistiques mises en œuvre par les États, ou par l'Europe (Gosse, 1997 ; Martel, 1997). Certaines langues pourraient alors bénéficier de politiques de mise en valeur tandis que certaines pourraient, à l'inverse, se retrouver mises à l'écart. Dans ce contexte, il convient de mentionner les récents discours du président du Parlement de la République slovaque (leader du SNS - Parti national slovaque, membre de la coalition gouvernementale) qui appelle de ses vœux un renforcement de la langue russe dans les établissements scolaires slovaques, souhaitant même que l'enseignement du russe devienne obligatoire à la place de l'anglais. Il serait néanmoins prématuré d'attendre que cet appel soit suivi favorablement par les apprenants et leurs parents au moment d'arrêter leur choix.

La définition d'une politique adéquate à l'enseignement des langues ne peut donc ignorer l'image des langues. Si les représentations sociales constituent une forme de transmission de pensée assez stable dans une société donnée, personne ne peut dire qu'elles n'évoluent pas avec la société qui les véhiculent.

Pour étayer notre recherche, nous avons opté pour des méthodes quantitatives notamment pour un questionnaire écrit distribué aux étudiants de la Faculté des sciences politiques et des relations internationales de 1'Université Matej Bel de Banská Bystrica en Slovaquie, soit environ 63 personnes ( 21 hommes et 42 femmes) âgés de 18 à 23 ans et inscrites en première (40) et deuxième année (23) de licence.

Le questionnaire se divise en deux parties. La première concerne directement l'apprentissage des langues, les choix effectués et les justifications de ceux-ci tout au long de la scolarité pré-universitaire (primaire et secondaire). Dans la seconde partie $\mathrm{du}$ questionnaire visant à déterminer l'image des langues, nous nous sommes notamment appuyés sur les travaux de Louise Dabène (1994) relatifs aux statuts informels des langues. Les langues retenues ont été l'anglais, l'allemand, le chinois, l'espagnol, le français et le russe. Cinq de ces six langues apparaissent la plupart du temps dans l'offre des établissements slovaques. Nous justifions l'ajout du chinois par l'expansion sans précédent de cette langue au niveau international.

L'origine géographique des répondants a été prise en considération : ce facteur nous a semblé susceptible d'influencer le choix des langues et les représentations sociales de celles-ci, car l'ouest de la Slovaquie se trouve à la frontière autrichienne et l'est du pays est situé à la frontière ukrainienne. Les répondants sont actuellement inscrits au programme "relations internationales », formation qui est unique en son genre dans l'espace académique slovaque car aucun autre établissement du pays ne propose de programme similaire, ce qui garantit une répartition géographique équilibrée. Les étudiants de ce programme de licence proviennent de toutes les régions de Slovaquie, phénomène moins fréquent dans les autres facultés de notre université, celles-ci ayant plutôt une portée régionale.

$36 \%$ des étudiants interrogés ont effectué leur scolarité primaire et secondaire en Slovaquie centrale, $25 \%$ en Slovaquie occidentale et $21 \%$ en Slovaquie orientale. 19 $\%$ des répondants n'étaient pas de nationalité slovaque (la plupart d'entre eux étaient 
Ukrainiens $)^{7}$ et ont donc réalisé leur cursus pré-universitaire dans un autre pays. Une grande partie des questions étant directement liée à enseignement primaire et secondaire en Slovaquie, nous avons choisi de ne pas tenir compte des réponses de ces étudiants lors de l'interprétation de la première partie des résultats du questionnaire. Nous avons en revanche considéré les réponses de ces apprenants comme intéressantes et importantes en dépouillant la seconde partie du questionnaire, consacrée aux représentations sociales des langues. Le nombre d'étudiants originaires d'Ukraine, voire d'autres pays d'Europe orientale, a sensiblement augmenté ces dernières années, il est donc indispensable que le système scolaire slovaque s'imprègne de cette réalité et ce à tous les niveaux d'enseignement.

Les réponses à la première partie du questionnaire démontrent que tous les étudiants slovaques ont jusqu'à présent étudié un minimum de deux langues étrangères - que ce soit à l'école ou en dehors. Pour d'évidentes raisons, nous avons ensuite observé l'appropriation des langues en milieu scolaire. De par la tranche d'âge des répondants, nous pouvons affirmer que la réforme curriculaire du Programme du Millénaire introduisant l'apprentissage de la première langue étrangère en troisième année d'école primaire et celle de la deuxième langue en septième année - a concerné l'ensemble des répondants.

Dans le cas de la première langue, la situation est limpide : $94 \%$ des personnes interrogées ont mentionné l'anglais comme première langue étrangère étudiée - $59 \%$ des répondants ont débuté leur apprentissage dès la première année d'école primaire. Concernant le choix de l'anglais, la quasi-totalité des répondants ont indiqué être satisfaits. Ces données indiquent incontestablement que la mise en place de l'anglais en tant que première langue obligatoire a constitué un ajustement inutile puisque cette langue était déjà choisie volontairement par la majorité des étudiants.

Une partie des répondants a également eu la possibilité - ou a décidé - d'apprendre une troisième langue et ce dès le second niveau de l'école primaire. L'offre comprenait l'ensemble des langues habituellement proposées (allemand, espagnol, français et russe) et la durée d'étude a varié selon les cas. Les troisièmes langues choisies ont été perçues comme satisfaisantes par les personnes interrogées, qui ne changeraient pas leur décision aujourd'hui.

La deuxième langue a bénéficié d'une attention particulière, car le Programme du Millénaire a rendu obligatoire son apprentissage à partir de la cinquième puis de la septième année d'école primaire mais aujourd'hui cette matière est seulement facultative. Les données recueillies nous permettent d'établir un ordre d'importance des secondes langues les plus apprises : la première étant l'allemand (56\%), suivi du russe $(19 \%)$ et de l'espagnol et du français, à égalité $(10 \%)$. Près de la moitié des répondants a changé de seconde langue en passant du primaire au secondaire, en dépit d'une satisfaction antérieure vis-à-vis de la deuxième langue étudiée jusqu'alors au niveau primaire.

Il est également nécessaire d'observer divers facteurs ayant influencé le choix des langues à étudier : pour ce qui est de la première langue - dans notre cas, l'anglais - la plupart des personnes interrogées indiquent avoir pris leur décisions seules éventuellement aidées par leurs parents ( $48 \%$ au total). Dans le cas de la deuxième langue, le facteur déterminant a été l'influence de l'école (48\%) la décision

${ }^{7}$ Principalement en première année de licence.

XLinguae, Volume 12 Issue 1XL, January 2019, ISSN 1337-8384, eISSN 2453-711X 
personnelle n'arrivant qu'en seconde position (33\%). D'autres facteurs tels que l'influence des parents, des frères et sœurs ou des amis ont aussi été cités à proportions égales même si leur pourcentage était bien moindre.

L'objectif de la seconde partie du questionnaire a été de cerner la perception de chaque langue par les étudiants quelles que soient les langues étudiées jusqu'à présent. La palette de réponses était la suivante : 1) Absolument d'accord, 2) Plutôt d'accord, 3) Plutôt pas d'accord et 4) Absolument pas d'accord. Un total de 11 affirmations a ainsi été évalué pour chaque langue. Nous avons regroupé ces affirmations au sein de 4 ensembles à analyser dans le cadre de nos recherches : 1) L'utilité (la maîtrise de cette langue est valorisée sur le marché du travail en Slovaquie / la maîtrise de cette langue est valorisée sur le marché du travail en Europe / la maîtrise de cette langue permet d'accéder aux programmes de mobilité étudiante proposés par mon établissement / j'associe cette langue au voyage), 2) Le caractère esthétique (j'apprécie la prononciation de cette langue et sa mélodie / j'apprécie la richesse culturelle associée à cette langue), 3) Le degré de difficulté (je considère cette langue comme difficile), 4) Le prestige (cette langue possède un statut important dans le cadre de l'Union européenne / cette langue possède un statut important à l'échelle internationale / cette langue est généralement très appréciée / cette langue permet d'obtenir une meilleure formation en Slovaquie).

Si la langue anglaise n'est pas considérée comme difficile par $71 \%$ des étudiants interrogés, les trois autres critères (utilité, caractère esthétique et prestige) ont été évalués positivement.

La perception de la langue allemande présente pour sa part une intéressante dualité : si elle est considérée comme un «plus » très utile en Slovaquie (83\%) et en Europe (98 $\%$ ), elle reste en général peu appréciée ( $76 \%$ d'évaluations négatives) et son aspect est jugé peu attirant (41\% de personnes ont exprimé un désaccord absolu quant à son aspect esthétique). La majorité des répondants a également estimé qu'il s'agissait d'une langue difficile (absolument d'accord : $59 \%$, plutôt d'accord : $29 \%$, soit près de $90 \%$ du total).

Bien que perçue comme étant la plus difficile dans le cadre de notre enquête (absolument d'accord : $56 \%$, plutôt d'accord : $35 \%$ ), la langue française est néanmoins jugée utile en Slovaquie (58\%), en Europe $(95 \%)$ et dans le monde (74\%). $45 \%$ des répondants estiment que l'étude du français permet d'accéder à une meilleure formation en Slovaquie et l'associent aux programmes de mobilité (62\%). L'aspect esthétique est également évalué de façon positive (77 \%).

Le caractère esthétique de l'espagnol a recueilli le plus de suffrages : $87 \%$ des étudiants se sont exprimés positivement sur cet aspect et $69 \%$ se sont déclarés intéressés par la richesse culturelle de cette langue. La langue de Cervantes est aussi généralement très appréciée $(90 \%)$ et sa maîtrise est considérée comme un atout sur le marché du travail européen $(67 \%)$ même si les répondants sont d'avis que l'espagnol s'avère peu utile en Slovaquie (74\%). $50 \%$ des personnes interrogées pensent que cette langue n'est pas difficile.

Le russe est perçu comme la langue la moins difficile (92\%) proposée dans les établissements scolaires slovaques. La faible utilité de cette langue slave sur le marché du travail local (26\%) est compensée par la bienveillance qu'elle peut engendrer ainsi que par sa position appréciable au niveau mondial. Le russe inspire néanmoins peu de possibilités de mobilité ( $75 \%$ d'avis contraires) et les étudiants ne l'associent pas non plus à l'Union européenne (75\%). 
Pour conclure, le chinois - qui est pour le moment proposé par très peu d'établissements - est considéré comme une langue difficile par $92 \%$ des répondants. Les étudiants reconnaissent en revanche son utilité au niveau international (83\%) même s'ils pensent que cette langue reste peu appréciée (77 \% d'avis contraires).

\section{Conclusion}

La politique linguistique de l'UE repose sur le respect de la diversité linguistique dans tous les États membres et sur la création d'un dialogue interculturel dans son ensemble. Afin de garantir le respect mutuel, l'Union encourage l'enseignement et l'apprentissage des langues étrangères et la mobilité des citoyens à travers des programmes consacrés à l'éducation et à la formation professionnelle.

Les compétences en langues étrangères jouent un rôle de plus en plus important pour renforcer la capacité d'insertion professionnelle des jeunes et les préparer à travailler sur le marché internationalisé de l'emploi. Elles constituent également un facteur de compétitivité : des compétences linguistiques faibles font perdre des marchés à de nombreuses entreprises et handicapent les travailleurs souhaitant trouver un emploi à l'étranger.

Si la diversité linguistique est bien ancrée dans l'histoire de la Slovaquie, cette réalité a été renforcée après l'adhésion du pays aux structures de l'Union européenne (le Programme du Millénaire introduisant une langue étrangère obligatoire et une langue optionnelle constitue en ce sens une étape capitale). Notre expérience pédagogique nous a amené à constater des changements assez importants dans l'apprentissage des langues durant la décennie qui a suivi l'adhésion de notre pays dans l'UE. Nous pouvons ici parler de «reconfiguration linguistique» en Slovaquie (avec un recul considérable des langues «traditionnelles» longtemps considérées comme prestigieuses en raison du contexte économique et européen - cet affaiblissement ayant profité à la langue russe) assortie d'un changement de statut des langues étrangères en présence (la deuxième langue étrangère cessant d'être obligatoire pour devenir facultative) et d'une baisse du niveau en langues étrangères chez les apprenants (si cette baisse de niveau est également observée dans le cas de la première langue étrangère, elle est bien plus évidente pour la deuxième langue).

Ces constats nous ont conduit à effectuer une recherche sur le choix et les motivations liés aux langues étrangères chez les apprenants slovaques tout en tenant compte des représentations que s'en font les locuteurs. L'enquête menée nous a permis de constater que les différentes langues présentes sur l'échiquier scolaire en Slovaquie ne sont pas traitées sur un pied d'égalité en raison de leur image qui joue un rôle dans le choix des élèves et de leurs parents en matière d'éducation linguistique. Si l'allemand et le français sont considérés par nos répondants comme importants pour le marché du travail en Slovaquie et en Europe, ces deux langues sont souvent synonymes de difficultés lors de leur apprentissage. Reste à savoir dans quelle mesure la facilité d'apprentissage peut s'avérer décisive lors du choix de la langue à étudier.

Les résultats présentés dans cette contribution sont loin de prétendre à l'exhaustivité et ils gagneraient à être complétés par des recherches ultérieures. Ce travail nous a néanmoins permis de cerner des éléments liés à l'image des langues auprès des étudiants en relations internationales, c'est-à-dire de personnes censées travailler, après leurs études supérieures dans un environnement plurilingue et pluriculturel. Notre recherche devra être enrichie par d'autres enquêtes - notamment auprès d'élèves du primaire et du secondaire qui doivent actuellement effectuer leurs choix, 
mais également auprès des parents afin de sonder les attentes de ceux-ci. Les opinions des enseignants et des responsables d'établissements constitueront un autre indicateur d'importance tout comme les remarques des entreprises étrangères et multinationales implantées dans notre pays.

$\mathrm{Si}$ les politiques linguistiques en Slovaquie visent à mettre en œuvre les recommandations européennes en termes de diversification des langues apprises et de construction de compétences plurilingues, elles devraient également tenir compte des réalités sur le marché du travail, ainsi que des représentations circulant dans la société à propos des langues, afin d'aboutir au développement d'organisations curriculaires et de démarches pédagogiques adaptées.

Les politiques éducatives pour l'enseignement des langues devraient être analysées dans un contexte plus large que celui relevant uniquement de la didactique ou de la pédagogie. Il convient de porter plus d'attention à la création de ces politiques, car elles impliquent une prise de décision et une négociation des acteurs individuels, puis la création de documents pertinents, l'adoption de stratégies ou encore le choix d'un mode de financement. Les acteurs individuels y jouent un rôle important, car ils se distinguent par leur position institutionnelle et diffèrent de par leurs intérêts, motivations et sources de financement ce qui entraîne également diverses possibilités d'influence.

Cet article fait partie du projet pédagogique en cours VEGA $n^{\circ} 1 / 0949 / 17$ «Le concept de "soft power" dans le contexte d'un environnement international en mutation et son potentiel pour les stratégies des petits États ».

\section{Bibliographic references}

AIDARBEK, A. - KANAT, Y. 2014. Education as a Soft Power Instrument of Foreign Policy. In: ScienceDirect, Procedia - Social and Behavioral Sciences, n. 143, pp. $501-503$.

BADINSKA, M. 2016. Critical reflexion as a tool in a process toward achieving intercultural communication competence and cultural intelligence. In: Kulturna inteligencia ako dolezity predpoklad konkurencieschopnosti Slovenska v globalnom prostredi. Banska Bystrica: Univerzita Mateja Bela, Ekonomicka fakulta, pp. 39-46. ISBN 978-80-557-1125-6.

BENCEKOVA, M. 2016. Frazeologia politologickych textov v kontexte kulturnej inteligencie. In: Od textu k prekladu XI. Praha: Jednota tlumocniku a prekladatelu, pp. 35-46. ISBN 978-80-7374-124-2.

BOCANOVA, M. - SLOBODOVA-NOVAKOVA, K. 2018. Addressing the issues of poverty and social exclusion of the city's inhabitants on principles based on Christianity. In: European Journal of Science and Theology, vol. 14, no. 4, pp. 85-93. ISSN 1841-0464.

COWAN, G. - ARSENAULT A. 2008. Moving from Monologue to Dialogue to Collaboration: The Three Layers of Public Diplomacy, The ANNALS of the American Academy of Political and Social Science 2008, pp. 10-30.

DABENE, L. 1994. Repcres sociolinguistiques pour l'enseignement des langues. Paris: Hachette.

DABENE, L. 1997. L'image des langues et leur apprentissage. In: Les langues et leurs images. Neuchatel: IRDP.

DEREGNAUCOURT, V. 2012. Apprendre une langue, le choix des parents: Entre heritage et pragmatisme, Editions universitaires europeennes. ISBN 978-3841789969. DOROZHKIN, E.M. - KALIMULLIN, A.M. - MIGACHEVA, G.N. - SOKOLOVA, T.B. 2018. Optimization of the Subject Matter of Profile Training Disciplines for Bachelors' Vocational Education on the Basis of Occupational Standards. In: 
EURASIA Journal of Mathematics, Science and Technology Education, vol. 14, n. 3, pp. 859-876. ISSN 13058215, 13058223

FIALA, P. - SCHUBERT, K. 2000. Moderni analyza politiky. Uvedeni do teorii a metod policy analysis. Brno: Barrister \& Principal.

FOWLER, F. C. 2004. Policy studies for educational leaders: An introduction. Upper Saddle River: Pearson Prentice Hall.

GOSSE, N. 1997. L'enseignement des langues : un enjeu europeen que se partagent essentiellement l'anglais et le français: Illustrations et explications à travers l'experience des programmes ERASMUS et LINGUA. In: Les langues et leurs images. Neuchatel: IRDP.

GURA, R. 2011. Niekolko poznamok k frankofonii ako integralnej sucasti kulturnej diplomacie Francuzskej republiky, jej postaveni a ulohach v strednej Europe na zaciatku 21. storocia. In: Brana jazykov k ludom otvorena. Pariz: Narodny institut vychodnych jazykov a kultur, pp. 50 - 74. ISBN 978-2-9536153-2-6.

GURA, R. - ROSTEKOVA, M. 2015. Mobilite et integration europeenne, l'exemple de la Slovaquie. In: EUROPEANA, n. 5, Les mobilites etudiantes europeennes dans le contexte international. Paris: L'Harmattan, pp. 17-41. ISBN 978-2-343-05805-4.

GURA, R. 2018. Makka moc najvplyvnejsich statov sveta. In: Makka moc a jej vyznam v medzinarodnych vztahoch v 21. storoci: teoreticke aspekty, regionalne kontexty. Banska Bystrica: Vydavatelstvo Univerzity Mateja Bela - Belianum, pp. 117-129. ISBN 978-80-557-1387-8.

HANESOVA, D. 2015. Plurilingualism - an educational challenge: the case of Slovakia. In: JoLaCE : journal of language and cultural education. Nitra: SlovakEdu, Vol. 3, n. 2, pp. 111-131. ISSN 1339-4045.

CHOVANCOVA, K. 2008. Interkulturalita ako "pridana hodnota" vo vyucovacom procese. In: Europa v skole. Nitra: Enigma, pp. 181-199. ISBN 978-80-89132-58-4.

KAMALOVA, L.A. - ZAKIROVA, V.G. 2014. Technique of comparative studying of the Russian and Tatar national fairy tales at modern elementary school in a context of dialogue of cultures. In: Middle - East Journal of Scientific Research, vol. 2, n. 1, pp. 33-37. ISSN 19909233, 19998147

KALICKY, J. - LASICOVA, J. 2017. Conservatism as a catalyst for economic changes in Central Europe states. MEST Journal, Vol. 5, n. 1. Belgrade: Faculty of Business and Law, pp. 1-7. ISSN 2334-705.

KHRULYOVA A.A. - SAKHIEVA R.G. 2017. Forming of Informational Culture as a Necessary Condition of the Level Raising of Higher Education. In: Man In India, vol. 97, n. 15, pp. 211-225. ISSN 00251569

KUBEKOVA, J. 2017. Interkomprehenzia ako vychodisko pre interkulturny dialog. In: Kulturna inteligencia ako dolezity predpoklad konkurencieschopnosti Slovenska v globalnom prostredi. Banska Bystrica: Vydavatelstvo Univerzity Mateja Bela Belianum, pp. 57-62. ISBN 978-80-557-1324-3.

KUBEKOVA, J. 2018. Jazykova politika a globalizacne tendencie v kontexte vyziev k plurilingvalnej a multikulturnej spolocnosti. Zbornik vedeckych studii III z projektu VEGA 1/0934/16. Kulturna inteligencia ako dolezity predpoklad konkurencieschopnosti Slovenska v globalnom prostredi. Banska Bystrica: Ekonomicka fakulta Univerzity Mateja Bela, pp. 61-71. ISBN 978-80-557-1488-2

LEVIN, B. 2008. How to change 5000 schools: A practical and positive approach for leading change at every level. Cambridge, MA: Harvard Education Press.

LI, N. - PYRKOVA, K.V. - RYABOVA, T.V. 2017. Teaching Communication Skills and Decision-Making to University Students. In: EURASIA Journal of Mathematics, Science and Technology Education, vol. 13, n. 8, pp. 4715-4723. ISSN 13058215,13058223

XLinguae, Volume 12 Issue 1XL, January 2019, ISSN 1337-8384, eISSN 2453-711X 
MARTEL, A. 1997. Quelques materiaux socioeconomiques dans l'architecture des representations en langues secondes et etrangcres. In: Les langues et leurs images. Neuchatel: IRDP.

MASALIMOVA, A.R. - LEVINA, E.Y. - PLATONOVA, R.I. - YAKUBENKO, K.Yu. - MAMITOVA, N.V. - ARZUMANOVA, L.L. - GREBENNIKOV, V.V. MARCHUK, N.N. 2017. Cognitive Simulation as Integrated Innovative Technology in Teaching of Social and Humanitarian Disciplines. In: EURASIA Journal of Mathematics, Science and Technology Education, vol. 13, n. 8, pp. 4915-4928. ISSN 13058215,13058223

MENTER, I. - VALEEVA, R. - KALIMULLIN, A. 2017. A tale of two countriesforty years on: politics and teacher education in Russia and England. In: European Journal of Teacher Education, vol. 40, n. 5, pp. 616-629. ISSN 14695928, 02619768

MESKOVA, L. 2016. Cultural differences between Western Europe and the former Eastern bloc. In: Culture and paradiplomatic identity: instruments in sustaining EU. Newcastle upon Tyne: Cambridge Scholars Publishing, pp. 1-15. ISBN 978-1-44388734-2.

MOLNAROVA. 2015. K otazke medialnej kompetencie buducich ucitelov nemeckeho jazyka. In: Aplikovane jazyky $\mathrm{v}$ univerzitnom kontexte - elektronicka podpora vzdelavania. Zvolen: Technicka univerzita vo Zvolene, pp. 75-82. ISBN 97880-228-2797-3.

MONDAVIO, A. 1997. L'image des langues dans le paysage socioeconomique hongrois. In: Les langues et leurs images. Neuchatel: IRDP.

NYE, J.S. 2009. Get Smart: Combining Hard and Soft Power. In: Foreign Affairs July 1, 2009, Available online: http://www.foreignaffairs.com/articles/65163/joseph-s-nye$\mathrm{jr} /$ getsmart?page $=2 \#>$

NYE, J. 2009. Smart Power, New Perspectives Quaterly, Vol. 26, n. 2, pp. 7-9.

PALOVA, M. - ZELENAKOVA, M. 2018. We Are Looking for Graduates with Professional Experience: Forum Students - Enterprises 2018 Offers a Solution to the Slovak Paradox. In: Politicke vedy. Vol. 21, N. 2, pp. 203 - 210. ISSN 1335 - 2741.

PETKOVSEK, R. 2014. Nasilje in etika kriza v luci eksistencialne analitike in mimeticne teorije. In: Bogoslovni vestnik, vol. 74, n.4, pp. 575-592. ISSN 1581-2987. RIZEKOVA, I. 2018. La preparation linguistique et socio-culturelle des etudiants slovaques à la mobilite en France. In: Gerflint. Synergies Europe n 13, pp. 59-67. ISSN 1951-6088.

ROSPUTINSKY, P. 2016. The participation of the European Union in international economic organizations. In: Globalization and its socio-economic consequences: proceedings of 16th international scientific conference. Rajecke Teplice, Zilina: University of Zilina, pp. 1880-1887. ISBN 978-80-8154-191-9.

ROSKOVA, M. 2006. Interkulturelle Aspekte im Prozess der Ubersetzung am Beispiel von Metaphern im Wirtschaftsdeutsch, In: Preklad a tlmocenie 7 : sociokulturne aspekty prekladu a tlmocenia: pritomnost a buducnost. Banska Bystrica: Univerzita Mateja Bela, pp. 215-223. ISBN 80-8083342-7.

ROSTEKOVA, M. 2018. Francuzsko-slovenska spolupraca ako motor internacionalizacie vysokoskolskeho vzdelavania na Slovensku. In: Politicke vedy, vol. 21, n. 1, Banska Bystrica: Fakulta politickych vied a medzinarodnych vztahov UMB, pp. $204-211$. ISSN 1335-274.

SCHMITT, F. 2014. Les representations sociales en comparaison interculturelle sur l'exemple d'une etude comparative des Français et des Slovaques. In: Foreign languages: a bridge to innovations in higher education. Banska Bystrica: Univerzita Mateja Bela v Banskej Bystrici, Ekonomicka fakulta, pp. 84-89. ISBN 978-80-5570756-3.

TEREM, P. 2011. Les changements sur la carte politilue de l'Europe centrale et leurs implications sur le profil de l'infrastructure educative slovaque. In : Ianua ad linguas hominesque reserata III, La porte des langues s'ouvre aux hommes III, Paris: Narodny 
institut vychodnych jazykov a kultur, Katedra strednej a vychodnej Europy, pp. 180197. ISBN 978-2-9536153-2-6.

TEREM, P. - CAJKA, P. - RYSOVA, L. 2015. Europe 2020 Strategy: evaluation, implementation, and prognoses for the Slovak Republic. In: Economics and Sociology, vol. 8, n. 2, pp. 154-171. ISSN 2071-789X.

VALCO, M. 2018. Sekularizacia ako vyzva pre tradicne nabozenstva Europy podla Charlesa Taylora [Secularization as a Challenge for Traditional Religions in Europe according to Charles Taylor]. In: Historia Ecclesiastica, vol. 9, n. 1, pp. 173-190. ISSN 1338-4341.

VESELY, A. 2013. Vzdelavaci politika: rozdilna vymezeni, predpoklady a implikace. Pedagogicka orientace, vol. 23, n. 3, pp. 279-297. ISBN 978-2-9536153-2-6.

VLCEK, D. 2018. Realizacia makkej moci v politike malych statov na zaciatku 21. storocia - niektore specifika. In: Makka moc a jej vyznam v medzinarodnych vztahoch v 21. storoci: teoreticke aspekty, regionalne kontexty. Banska Bystrica: Vydavatelstvo Univerzity Mateja Bela - Belianum, pp. 9-18. ISBN 978-80-557-1387-8.

Vzdelavaci system v Slovenskej republike, 2018. UIPS. Available online: http://web.uips.sk/download/rs/Vzdelavaci_system_v_Slovenskej_Republike.pdf WHELAN, F. 2009. Lessons learned: How good policies produce better schools. London: Fenton Whelan.

ZAZRIVCOVA, M. 2014. Interaction culturelles franco-slovaques au XXe siccle.

In: Tandem linguistique et immersion. Cluj-Napoca: Editura Medicala Universitara Iuliu Hatieganu, ISBN 978-973-693-604-3.

Words: 8196

Characters : 55675 (30,93 standard pages)

PaedDr. Mária Rošteková, PhD.

Department of International Relations and Diplomacy

Faculty of Political Science and International Relations

Matej Bel University in Banská Bystrica

Kuzmányho 1

97401 Banská Bystrica

Slovakia

XLinguae, Volume 12 Issue 1XL, January 2019, ISSN 1337-8384, eISSN 2453-711X 